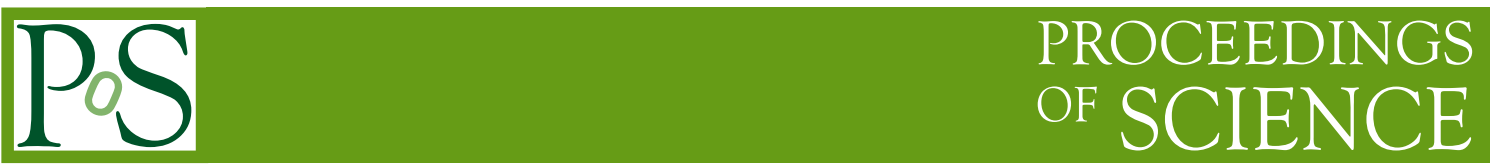

\title{
Higgs production at the Tevatron: predictions and uncertainties
}

\author{
Julien Baglio* \\ Laboratoire de Physique Théorique, Université Paris-Sud 11 et CNRS, F-91405 Orsay (France) \\ E-mail: Julien.Baglio@th.u-psud.fr
}

\begin{abstract}
We present an update of the theoretical predictions for the production cross sections of the Standard Model Higgs boson at the Tevatron collider in the gluon-gluon fusion channel, including all relevant higher order QCD and electroweak corrections in perturbation theory. A thorough analysis of the theoretical uncertainties affecting these predictions will then follow: the scale uncertainty, the uncertainties associated with the PDF and the errors on the value of the strong coupling $\alpha_{S}$ and the uncertainties related to the use of an effective field theory in the gluon-gluon channel. The combined theoretical uncertainty is found to be large and will impact the Tevatron Higgs exclusion bound.
\end{abstract}

35th International Conference of High Energy Physics - ICHEP2010,

July 22-28, 2010

Paris France

${ }^{*}$ Speaker. 


\section{Introduction}

The quest for the Higgs boson [1,2], the relic of the electroweak (EW) symmetry breaking in the Standard Model, is one of the key searches at the current Fermilab Tevatron and CERN LHC colliders. Whereas the LHC is still awaiting for at least $1 \mathrm{fb}^{-1}$ of data to have significant results, the Tevatron experiments are already sensitive to a Higgs signal at masses $M_{H} \approx 165 \mathrm{GeV}$ [3].

The two main search channels at the Tevatron are the gluon-gluon fusion, $g g \rightarrow H$, known up to next-to-next-to-leading-order (NNLO) in QCD in the infinite $M_{t}$ approximation $[4,5,6$, 7, 14, 15] with mixed NNLO QCD+EW corrections [8, 9], followed by $H \rightarrow W W^{*}$ decay, and the Higgs-strahlung process $q \bar{q} \rightarrow H V$ known up to NNLO in QCD [10, 11, 12] with NLO EW corrections[13], followed by $H \rightarrow b \bar{b}$ decay.

In this talk we discuss an update of the theoretical prediction for the production cross sections [18] and a detailed analysis of the uncertainties which affect them: the scale uncertainties due to missing QCD higher-order terms, the PDF $+\alpha_{s}$ uncertainties taking into account a theoretical uncertainty on $\alpha_{s}$ and the uncertainties due to the use of an effective field theory approach.

We will focus on the $g g \rightarrow H$ process for which the sensitivity at the Tevatron is the highest and which allowed the CDF and D0 collaboration to set exclusion bounds for the Higgs boson in the mass range $M_{H}=158-175 \mathrm{GeV}$ [3]. A detailed discussion of the Higgs-strahlung process, which is relevant a lower Higgs masses, can be found in Ref. [18].

\section{Theoretical predictions and uncertainties in the gg-fusion channel}

The starting point of our calculation of the $g g \rightarrow H$ cross section is the Fortran program HIGLU [16] in which we have made modifications in order to incorporate the NNLO QCD and the electroweak corrections. The cross section is evaluated at the central value $\mu_{R}=\mu_{F}=M_{H}$ for the renormalisation scale $\mu_{R}$ which defines the strong coupling constant $\alpha_{s}$ and on the factorisation scale $\mu_{F}$ at which the matching between the partonic calculation and the non-perturbative parton distribution functions (PDF) terms is done. This choice, contrary to the choice $\mu_{R}=\mu_{F}=\frac{1}{2} M_{H}$, does not allow to indirectly include the soft-gluon resumation contributions which slightly increase the cross section [14]. A detailed discussion for this more suitable scale choice can be found in the addendum of Ref. [18]. For the parton densities, the latest set of NNLO MSTW PDFs [19] will be used but other sets will be discussed too.

The theoretical uncertainties are included as follows.

\section{a. Higher orders and scale variation}

In a perturbative calculation, the series is truncated, which implies that the cross sections are dependent on the renormalisation scale $\mu_{R}$ and on the factorisation scale $\mu_{F}$. The uncertainty obtained by the variation of the two scales is taken as an estimate of the unknown higher-order terms and is in general the dominant source of uncertainties. Starting with the median scale $\mu_{0}=M_{H}$ for which the central prediction is obtained, the two scales $\mu_{R}, \mu_{F}$ are varied within the interval $\mu_{0} / \kappa \leq \mu_{R}, \mu_{F} \leq \kappa / \mu_{0}$, with a chosen value $\kappa=2,3,4$, etc... In order to make a suitable choice of the $\kappa$ value, we compare $\sigma^{\mathrm{NLO}}$ with the central $\sigma^{\mathrm{NNLO}}$ and we require the error band on the NLO results to catch the latter cross section. As seen on Fig. 1 (left) we need at least $\kappa=3$ according to this procedure. 

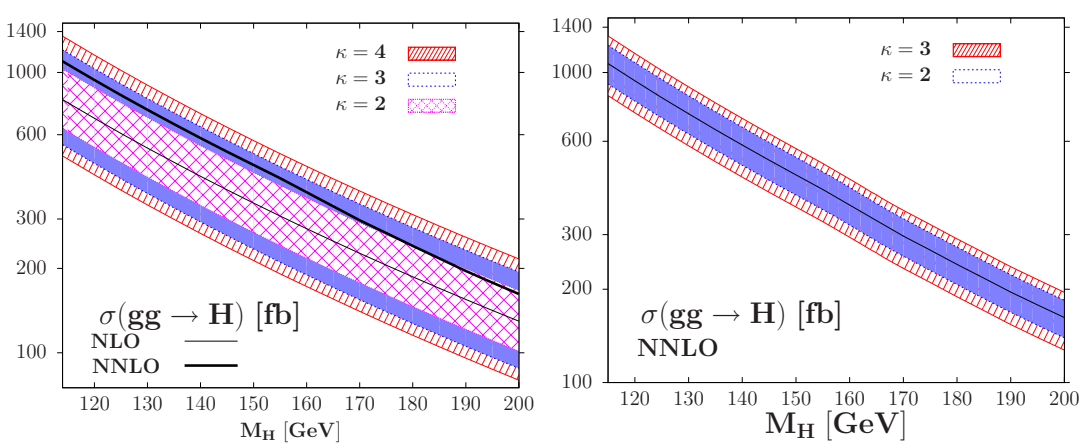

Figure 1: Left: the scale dependence of $\sigma^{\mathrm{NLO}}(g g \rightarrow H)$ at the Tevatron as a function of $M_{H}$ for variations $M_{H} / \kappa \leq \mu_{R}, \mu_{F} \leq \kappa M_{H}$ with $\kappa=2,3.4$ compared to $\sigma^{\mathrm{NNLO}}$ evaluated at the central scale $\mu_{R}=\mu_{F}=M_{H}$. Right: the scale uncertainty bands of $\sigma^{\mathrm{NNLO}}$ for a variation in the domain $M_{H} / \kappa \leq \mu_{R}, \mu_{F} \leq \kappa M_{H}$ with $\kappa=2,3$.

Using this range for $\mu_{R}, \mu_{F}$ for the NNLO cross section we obtain a scale variation of $\Delta^{\text {scale }} \sigma \simeq$ $20 \%$ for the relevant range of $M_{H}$ values, nearly twice the much smaller variation $\Delta^{\text {scale }} \sigma \simeq 10-$ $12 \%$ adopted by the $\mathrm{CDF} / \mathrm{D} 0$ collaborations.

\section{b. The PDFs and $\alpha_{s}$ uncertainties}

The second most important source of uncertainties in the $g g \rightarrow H$ mechanism comes from the PDFs uncertainties together with the experimental and theoretical errors on the strong coupling constant $\alpha_{s}$. We estimate the PDF uncertainties with the help of the $2 \times 20$ PDF sets errors provided not only by the the MSTW collaboration [19], but also by the CTEQ [20] or ABKM [21] collaborations. We take into accounts the spread of both uncertainties and the central values obtained within these different sets. The calculation gives a 5-10\% error within all sets, but the ABKM central value is $\sim 25 \%$ smaller than the CTEQ/MSTW central values as can be seen in Fig. 6 of Ref. [18].

In addition to the PDF uncertainties, the errors coming from the theoretical and experimental uncertainties in the determination of the $\alpha_{s}$ value are considered. In the MSTW scheme we have $\alpha_{s}\left(M_{Z}^{2}\right)=0.1171_{-0.0034}^{+0.0034}(90 \% \mathrm{CL})$ at NNLO, and there is also a theoretical uncertainty estimated by the MSTW collaboration [19] as $\Delta^{\text {th }} \alpha_{s}=0.002$ at most at NNLO. We have computed the correlated PDF $+\Delta^{\exp } \alpha_{s}$ uncertainties using the MSTW set-up [22], and used the central fixed- $\alpha_{s}$ MSTW PDF set for $\Delta^{\text {th }} \alpha_{S}$. The result is shown in Fig. 7 of Ref. [18]; with only the experimental errors on $\alpha_{S}$, the MSTW/CTEQ and ABKM predictions cannot yet be reconciled, but this can be achieved with the addition of the uncertainty coming from the theoretical error on $\alpha_{S}$. We obtain a much larger uncertainty of $\simeq 20 \%$ compared to that of the $\sim 10 \%$ error obtained when using the $\mathrm{PDF}$ error only, as assumed by the CDF/D0 collaborations.

\section{c. The use of an effective theory approach}

The final set of uncertainties is specific to the gluon-gluon fusion mechanism which at NNLO is evaluated in an effective theory (EFT) approach where $M_{Q} \gg M_{H}$ for the contribution of a quark $Q$ in the loop. It is very accurate for the top-quark loop with a below $0.1 \%$ correction with finite top-mass effects for $M_{H} \lesssim 300 \mathrm{GeV}$ [23], but not for the b-quark loop where at NLO its omission leads to $\mathrm{a} \simeq 10 \%$ difference compared to the exact case. 
In order to estimate the error of ignoring the $b$-loop contribution at NNLO, we rescale the difference calculated at NLO where the exact result is known by the relative NLO/NNLO $K$-factors, $\Delta_{\mathrm{NNLO}}^{\mathrm{b}-\text { loop }}=\frac{\sigma_{\mathrm{exact}}^{\mathrm{NLO}}-\sigma_{\mathrm{EFT}}^{\mathrm{NLO}}}{\sigma_{\mathrm{exact}}^{\mathrm{NLO}}} \times \frac{K_{\mathrm{NLO}}}{K_{\mathrm{NNLO}}}$. This gives a $1-2 \%$ uncertainty. We then add a small uncertainty which is related to the difference between the on-shell bottom mass $M_{b}=4.75 \mathrm{GeV}$ and the mass in the $\overline{\mathrm{MS}}$ scheme, $m_{b}\left(m_{b}\right)=4.23 \mathrm{GeV}$; this amounts to $\sim 1-2 \%$ uncertainty in the b-loop contribution, leading to a total error of a few $\%$ as shown in Fig. 4 of Ref. [18].

The last EFT error is related to the mixed QCD-EW electroweak corrections which have been calculated with $M_{W / Z} \gg M_{H}$ [9]. As it is obviously not the case in practice, we should be cautious in using this (small) correction and assign an error which is of the same size by comparing it with the use of the exact NLO EW corrections only: $\Delta_{\mathrm{EW}}=\left(\sigma^{\mathrm{NNLOQCD}-\mathrm{EW}}-\sigma^{\mathrm{NLO}}\right) / \sigma^{\mathrm{NNLOQCD}-\mathrm{EW}}$. This gives an error of 3.5\% at most as shown in Fig. 5 of Ref. [18].

\section{Total uncertainties in both channels and conclusion}

The very important issue that remains is how to combine the various theoretical uncertainties on the cross section discussed in the previous section. The CDF collaboration adds quadratically the scale variation (with $\kappa=2$ ) with the PDF-error only, leading to a a $12 \%$ (scale) $\oplus 10 \%(\mathrm{PDF})=$ $16 \%$ total error, while D0 collaboration assumes a smaller error of $10 \%$. As these are theoretical errors, we believe that such a combination is not adequate. On the other hand, adding the errors linearly may appear to be too conservative. We thus propose a procedure which, to our opinion, is more reasonable: one calculates the maximal/minimal cross sections with respect to the scale variation, and apply on these cross sections the $\mathrm{PDF}+\Delta \alpha_{s}$ analysis in quadrature, with a final linear addition of the small EFT errors.
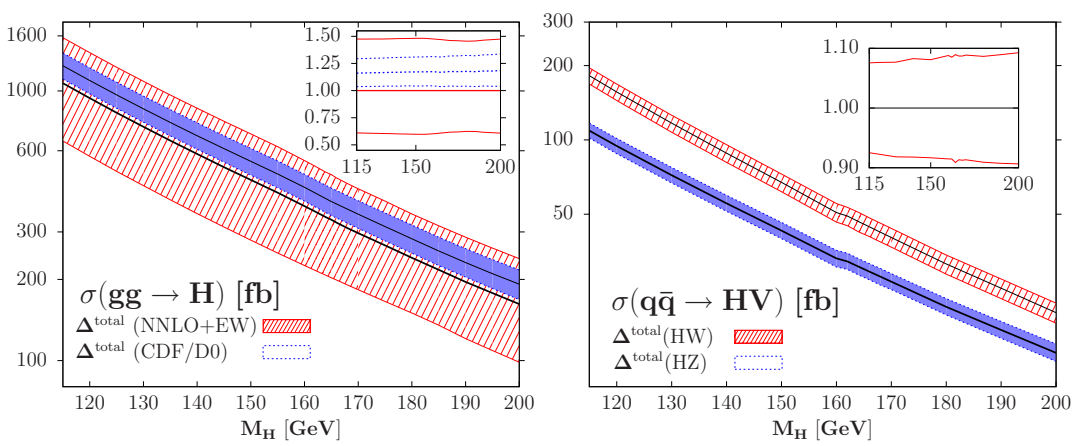

Figure 2: Left: the production cross section $\sigma(g g \rightarrow H)$ at NNLO at the Tevatron with the uncertainty band when all the uncertainties are added using our procedure. It is compared to $\sigma(g g \rightarrow H)$ at NNLL when the scale and PDF errors given in Ref. [17] are added in quadrature. In the insert the relative deviations are shown when the central values are normalized to $\sigma^{\mathrm{NNLO}+\mathrm{EW}}$. Right: the same but for the $q \bar{q} \rightarrow H V$ production channel.

We then obtain a total $\simeq \pm 40 \%$ uncertainty for the cross section in the gluon-gluon fusion mechanism $g g \rightarrow H$ in the entire $M_{H}$ range that is relevant at the Tevatron; Fig. 2 (left). This error is thus much larger than the $\simeq 10-15 \%$ uncertainty obtained in the CDF/D0 analysis [3]. This means that $\sigma_{\mathrm{gg} \rightarrow \mathrm{H}}^{\mathrm{NNLO}}$ could be a factor or two lower than what is assumed in the $p \bar{p} \rightarrow H \rightarrow W^{(*)} W^{(*)} \rightarrow \ell \ell v v$ analysis and that the 95\% CL CDF/D0 exclusion band $158 \leq M_{H} \leq 175 \mathrm{GeV}$ should then be reconsidered in the light of these large uncertainties. 
The same analysis has been performed for the $q \bar{q} \rightarrow H V$ channel and gives a total $\simeq \pm 8 \%$ uncertaintly, dominated by the PDF $+\alpha_{S}$ uncertainties. This is much more under control than for the gluon-gluon fusion channel, and twice the errors assumed by the CDF/D0 collaborations.

Acknowledgments JB would like to thank the ICHEP 2010 organisers for having accepted this presentation. This work is supported by the European network HEPTOOLS.

\section{References}

[1] P. Higgs, Phys. Lett. 12 (1964) 132; F. Englert and R. Brout, Phys. Rev. Lett. 13 (1964) 321; G. Guralnik, C. Hagen and T. Kibble, Phys. Rev. Lett. 13 (1964) 585.

[2] For a review, see: A. Djouadi, Phys. Rept. 457 (2008) 1.

[3] The CDF and D0 collaborations, arXiv:1007.4587.

[4] H. Georgi, S. Glashow, M. Machacek and D. Nanopoulos, Phys. Rev. Lett. 40 (1978) 692.

[5] A. Djouadi, M. Spira and P. Zerwas, Phys. Lett. B264 (1991) 440; S. Dawson, Nucl. Phys. B359 (1991) 283.

[6] M. Spira, A. Djouadi, D. Graudenz and P.M. Zerwas, Nucl. Phys. B453 (1995) 17.

[7] R.V. Harlander and W. Kilgore, Phys. Rev. Lett. 88 (2002) 201801; C. Anastasiou and K. Melnikov, Nucl. Phys. B646 (2002) 220; V. Ravindran, J. Smith and W.L. Van Neerven, Nucl. Phys. B665 (2003) 325.

[8] S. Actis, G. Passarino, C. Sturm and S. Uccirati, Nucl. Phys. B811 (2009) 182.

[9] C. Anastasiou, R. Boughezal and F. Pietriello, JHEP 0904 (2009) 003.

[10] S.L. Glashow, D.V. Nanopoulos and A. Yildiz, Phys. Rev. D18 (1978) 1724.

[11] G. Altarelli, R.K. Ellis and G. Martinelli, Nuc. Phys. B157 (1979) 461; J. Kubar-André and F. Paige, Phys. Rev. D19 (1979) 221; T. Han and S. Willenbrock, Phys. Lett. B273 (1991) 167; J. Ohnemus and W. J. Stirling, Phys. Rev. D47 (1993) 2722; M. Spira, Fortschr. Phys. 46 (1998) 203; A. Djouadi and M. Spira, Phys. Rev. D62 (2000) 014004.

[12] O. Brein, A. Djouadi and R. Harlander, Phys. Lett. B579 (2004) 149.

[13] M. L. Ciccolini, S. Dittmaier and M. Krämer, Phys. Rev. D68 (2003) 073003.

[14] S. Catani, D. de Florian, M. Grazzini and P. Nason, JHEP 0307 (2003) 028.

[15] C. Anastasiou et al., JHEP 0908 (2009) 099.

[16] The Fortran codes can be found in Michael Spira's web page, http://people.web.psi.ch/ mspira/. For HIGLU, see hep-ph/9510347.

[17] D. de Florian and G. Grazzini, Phys. Lett. B674 (2009) 291.

[18] J.Baglio and A. Djouadi, JHEP 1010 (2010) 064.

[19] A.D. Martin, W. Strirling, R. Thorne and G. Watt, Eur. Phys. J. C63 (2009) 189.

[20] P.M. Nadolsky et al. (CTEQ coll.), Phys. Rev. D78 (2008) 013004.

[21] S. Alekhin, J. Blumlein, S. Klein and S. Moch, arXiv:0908.2766.

[22] A.D. Martin, W. Strirling, R. Thorne and G. Watt, Eur. Phys. J. C64 (2009) 653.

[23] R. Harlander and K. Ozeren, arXiv:0909.3420; A. Pak, M. Rogal and M. Steinhauser, arXiv:0911.4662. 